\title{
ON NEW SUBCLASSES \\ OF BI-UNIVALENT FUNCTIONS ASSOCIATED WITH AL-OBOUDI DIFFERENTIAL OPERATOR
}

\author{
Amol B. Patil ${ }^{1}$, Uday H. Naik ${ }^{2}$ \\ ${ }^{1}$ Department of First Year Engineering \\ AISSMS's, College of Engineering \\ Pune, 411001, INDIA \\ ${ }^{2}$ Department of Mathematics \\ Willingdon College \\ Sangli, 416415, INDIA
}

\begin{abstract}
In the present paper we introduce two new subclasses of the class of bi-univalent functions defined on the open unit disk $\mathbb{U}$, which are associated with the Al-Oboudi differential operator. Also we obtain the estimates of the coefficients $\left|a_{2}\right|$ and $\left|a_{3}\right|$ for the functions of these classes. Relavent connections with the various well known results are indicated.
\end{abstract}

AMS Subject Classification: 30C45, 30C50

Key Words: Analytic function, Bi-univalent function, Al-Oboudi differential operator.

\section{Introduction}

Let $\mathcal{A}$ denote the class of all functions of the form

$$
f(z)=z+\sum_{k=2}^{\infty} a_{k} z^{k},
$$

which are analytic in the open unit disk,

\footnotetext{
Received: $\quad$ April 29, 2016

Revised: $\quad$ August 28, 2016

Published: October 21, 2016

(c) 2016 Academic Publications, Ltd. url: www.acadpubl.eu

${ }^{\S}$ Correspondence author
} 


$$
\mathbb{U}=\{z: z \in \mathbb{C},|z|<1\} .
$$

Let $\mathcal{S}$ denote the subclass of $\mathcal{A}$ consisting of the functions which are also univalent in $\mathbb{U}$ (for details, see [8]). It is well known that every function $f \in \mathcal{S}$ has an inverse $f^{-1}$, defined by

$$
f^{-1}(f(z))=z, \quad(z \in \mathbb{U})
$$

and

$$
f\left(f^{-1}(w)\right)=w, \quad\left(|w|<r_{0}(f), r_{0}(f) \geq \frac{1}{4}\right),
$$

where,

$$
\begin{aligned}
g(w) & =f^{-1}(w) \\
& =w-a_{2} w^{2}+\left(2 a_{2}^{2}-a_{3}\right) w^{3}-\left(5 a_{2}^{3}-5 a_{2} a_{3}+a_{4}\right) w^{4}+\cdots
\end{aligned}
$$

A function $f(z) \in \mathcal{A}$ is said to be bi-univalent in $\mathbb{U}$ if both $f(z)$ and $f^{-1}(z)$ are univalent in $\mathbb{U}$. We denote by $\Sigma$ the class of all bi-univalent functions in $\mathbb{U}$. The study of coefficient problems involving bi-univalent functions was revived recently by Srivastava et. al. [17]. Various subclasses of the bi-univalent function class $\Sigma$ were introduced and non-sharp estimates of $\left|a_{2}\right|$ and $\left|a_{3}\right|$ of the functions in these subclasses were found in some recent investigations (see $[5,6,7,9,11,14,16]$ etc.). For the brief history and examples of functions in the class $\Sigma$, see [17].

For functions in the class $\Sigma$, Lewin [10] proved that $\left|a_{2}\right|<1.51$, Brannan and Clunie [2] conjectured that $\left|a_{2}\right| \leq \sqrt{2}$ and Netanyahu [12] proved that $\max _{f \in \Sigma}\left|a_{2}\right|=4 / 3$. However the coefficient estimate problem for each $\left|a_{n}\right|$, $(n=3,4, \cdots)$ is still an open problem.

Brannan and Taha [4], (see also $[3,18]$ ) introduced the following two subclasses of the bi-univalent function class $\Sigma$ and obtained non-sharp estimates on the first two Taylor - Maclaurin coefficients $\left|a_{2}\right|$ and $\left|a_{3}\right|$ of functions in each of these subclasses.

Definition 1. [4] A function $f(z)$ given by (1) is said to be in the class $\mathcal{S}_{\Sigma}^{*}[\alpha]$ where $0<\alpha \leq 1$ if the following conditions are satisfied:

$$
f \in \Sigma, \quad\left|\arg \left\{\frac{z f^{\prime}(z)}{f(z)}\right\}\right|<\frac{\alpha \pi}{2}, \quad(z \in \mathbb{U})
$$


and

$$
\left|\arg \left\{\frac{w g^{\prime}(w)}{g(w)}\right\}\right|<\frac{\alpha \pi}{2}, \quad(w \in \mathbb{U})
$$

where the function $g$ is defined by (2).

The subclass $\mathcal{S}_{\Sigma}^{*}[\alpha]$ is known as the class of strongly bi-starlike functions of order $\alpha$.

Definition 2. [4] A function $f(z)$ given by (1) is said to be in the class $\mathcal{S}_{\Sigma}^{*}(\beta)$ where $0 \leq \beta<1$ if the following conditions are satisfied:

$$
f \in \Sigma, \quad \Re\left\{\frac{z f^{\prime}(z)}{f(z)}\right\}>\beta, \quad(z \in \mathbb{U})
$$

and

$$
\Re\left\{\frac{w g^{\prime}(w)}{g(w)}\right\}>\beta, \quad(w \in \mathbb{U})
$$

where the function $g$ is defined by $(2)$.

The subclass $\mathcal{S}_{\Sigma}^{*}(\beta)$ is known as the class of bi-starlike functions of order $\beta$.

For $f \in \mathcal{A}$, Al-Oboudi [1] introduced the following operator :

$$
\begin{gathered}
D_{\delta}^{0} f(z)=f(z) \\
D_{\delta}^{1} f(z)=(1-\delta) f(z)+\delta z f^{\prime}(z)=D_{\delta} f(z) ; \quad(\delta \geq 0) \\
D_{\delta}^{n} f(z)=D_{\delta}\left(D_{\delta}^{n-1} f(z)\right) ; \quad(n \in \mathbb{N})
\end{gathered}
$$

If $f$ is given by (1) then from (3) and (4) we see that,

$$
D_{\delta}^{n} f(z)=z+\sum_{k=2}^{\infty}[1+(k-1) \delta]^{n} a_{k} z^{k} ; \quad\left(n \in \mathbb{N}_{0}\right)
$$

with $D_{\delta}^{n} f(0)=0$ and when $\delta=1$, we get the Sălăgean's differential operator $D_{1}^{n}=D^{n}[15]$.

The object of the present paper is to introduce two new subclasses of the function class $\Sigma$ defined by using Al-Oboudi differential operator and to find estimates on the coefficients $\left|a_{2}\right|$ and $\left|a_{3}\right|$ for functions in these new subclasses. Firstly, in order to prove our main results, we need the following lemma [13]. 
Lemma 3. [13] If $p(z) \in \mathcal{P}$, the class of functions analytic in $\mathbb{U}$ with

$$
\Re(p(z))>0,
$$

then $\left|c_{n}\right| \leq 2$ for each $n \in \mathbb{N}$, where

$$
p(z)=1+c_{1} z+c_{2} z^{2}+c_{3} z^{3}+\cdots, \quad(z \in \mathbb{U}) .
$$

\section{Coefficient Bounds for the Function Class $\mathcal{B}_{\Sigma}(\delta, n, \alpha, \lambda)$}

Definition 4. A function $f(z)$ given by (1) is said to be in the class $\mathcal{B}_{\Sigma}(\delta, n, \alpha, \lambda)$ if the following conditions are satisfied:

$$
\begin{gathered}
f \in \Sigma, \quad\left|\arg \left\{\frac{(1-\lambda) D_{\delta}^{n} f(z)+\lambda D_{\delta}^{n+1} f(z)}{z}\right\}\right|<\frac{\alpha \pi}{2} \\
\left(0<\alpha \leq 1, \delta \geq 0, \lambda \geq 1, n \in \mathbb{N}_{0}, z \in \mathbb{U}\right)
\end{gathered}
$$

and

$$
\begin{gathered}
\left|\arg \left\{\frac{(1-\lambda) D_{\delta}^{n} g(w)+\lambda D_{\delta}^{n+1} g(w)}{w}\right\}\right|<\frac{\alpha \pi}{2} \\
\left(0<\alpha \leq 1, \delta \geq 0, \lambda \geq 1, n \in \mathbb{N}_{0}, w \in \mathbb{U}\right)
\end{gathered}
$$

where the function $g$ is given by (2).

We note that for $\delta=1$ the class $\mathcal{B}_{\Sigma}(\delta, n, \alpha, \lambda)$ reduces to the class $\mathcal{B}_{\Sigma}(n, \alpha, \lambda)$ introduced by S. Porwal and M. Darus [14] and for $\delta=1, n=0, \lambda=1$ the class $\mathcal{B}_{\Sigma}(\delta, n, \alpha, \lambda)$ reduces to the class $\mathcal{H}_{\Sigma}^{\alpha}$ introduced by Srivastava et.al. [17]. Also for $\delta=1, n=0$ this class reduces to the class $\mathcal{B}_{\Sigma}(\alpha, \lambda)$ introduced by B.A. Frasin and M.K. Aouf [9].

Theorem 5. Let the function $f(z)$ given by (1) be in the class $\mathcal{B}_{\Sigma}(\delta, n, \alpha, \lambda)$. Then,

$$
\left|a_{2}\right| \leq \frac{2 \alpha}{\sqrt{2 \alpha(1+2 \delta)^{n}(1+2 \lambda \delta)-(\alpha-1)(1+\delta)^{2 n}(1+\lambda \delta)^{2}}}
$$

and

$$
\left|a_{3}\right| \leq \frac{4 \alpha^{2}}{(1+\delta)^{2 n}(1+\lambda \delta)^{2}}+\frac{2 \alpha}{(1+2 \delta)^{n}(1+2 \lambda \delta)} .
$$


Proof. From (5) and (6), we have

$$
\frac{(1-\lambda) D_{\delta}^{n} f(z)+\lambda D_{\delta}^{n+1} f(z)}{z}=[p(z)]^{\alpha}
$$

and

$$
\frac{(1-\lambda) D_{\delta}^{n} g(w)+\lambda D_{\delta}^{n+1} g(w)}{w}=[q(w)]^{\alpha}
$$

where $p(z), q(w) \in \mathcal{P}$ with

$$
p(z)=1+c_{1} z+c_{2} z^{2}+c_{3} z^{3}+\cdots,(z \in \mathbb{U})
$$

and

$$
q(w)=1+d_{1} w+d_{2} w^{2}+d_{3} w^{3}+\cdots,(w \in \mathbb{U}) .
$$

Equating the coefficients in (9) and (10), we get

$$
\begin{gathered}
(1+\delta)^{n}(1+\lambda \delta) a_{2}=\alpha c_{1} \\
(1+2 \delta)^{n}(1+2 \lambda \delta) a_{3}=\alpha c_{2}+\frac{\alpha(\alpha-1)}{2} c_{1}^{2} \\
-(1+\delta)^{n}(1+\lambda \delta) a_{2}=\alpha d_{1} \\
(1+2 \delta)^{n}(1+2 \lambda \delta)\left(2 a_{2}^{2}-a_{3}\right)=\alpha d_{2}+\frac{\alpha(\alpha-1)}{2} d_{1}^{2}
\end{gathered}
$$

From (13) and (15), we obtain

$$
c_{1}=-d_{1}
$$

and

$$
2(1+\delta)^{2 n}(1+\lambda \delta)^{2} a_{2}^{2}=\alpha^{2}\left(c_{1}^{2}+d_{1}^{2}\right)
$$

Adding (14) and (16) and then using (18), we obtain

$$
a_{2}^{2}=\frac{\alpha^{2}\left(c_{2}+d_{2}\right)}{2 \alpha(1+2 \delta)^{n}(1+2 \lambda \delta)-(\alpha-1)(1+\delta)^{2 n}(1+\lambda \delta)^{2}}
$$

Using Lemma 3, we get

$$
a_{2}^{2} \leq \frac{4 \alpha^{2}}{2 \alpha(1+2 \delta)^{n}(1+2 \lambda \delta)-(\alpha-1)(1+\delta)^{2 n}(1+\lambda \delta)^{2}}
$$

which gives the desired result (7). 
Next, subtracting (16) from (14) and then using (17), we obtain

$$
a_{3}-a_{2}^{2}=\frac{\alpha\left(c_{2}-d_{2}\right)}{2(1+2 \delta)^{n}(1+2 \lambda \delta)}
$$

By (18), we get

$$
a_{3}=\frac{\alpha^{2}\left(c_{1}^{2}+d_{1}^{2}\right)}{2(1+\delta)^{2 n}(1+\lambda \delta)^{2}}+\frac{\alpha\left(c_{2}-d_{2}\right)}{2(1+2 \delta)^{n}(1+2 \lambda \delta)}
$$

Using Lemma 3, we get

$$
\left|a_{3}\right| \leq \frac{4 \alpha^{2}}{(1+\delta)^{2 n}(1+\lambda \delta)^{2}}+\frac{2 \alpha}{(1+2 \delta)^{n}(1+2 \lambda \delta)}
$$

which is the desired result (8).

This completes the proof of Theorem 5 .

\section{Coefficient Bounds for the Function Class $\mathcal{H}_{\Sigma}(\delta, n, \beta, \lambda)$}

Definition 6. A function $f(z)$ given by (1) is said to be in the class $\mathcal{H}_{\Sigma}(\delta, n, \beta, \lambda)$ if the following conditions are satisfied:

$$
\begin{gathered}
f \in \Sigma, \quad \Re\left\{\frac{(1-\lambda) D_{\delta}^{n} f(z)+\lambda D_{\delta}^{n+1} f(z)}{z}\right\}>\beta \\
\left(0 \leq \beta<1, \delta \geq 0, \lambda \geq 1, n \in \mathbb{N}_{0}, z \in \mathbb{U}\right)
\end{gathered}
$$

and

$$
\begin{aligned}
& \Re\left\{\frac{(1-\lambda) D_{\delta}^{n} g(w)+\lambda D_{\delta}^{n+1} g(w)}{w}\right\}>\beta \\
& \left(0 \leq \beta<1, \delta \geq 0, \lambda \geq 1, n \in \mathbb{N}_{0}, w \in \mathbb{U}\right),
\end{aligned}
$$

where the function $g$ is given by (2).

We note that for $\delta=1$ the class $\mathcal{H}_{\Sigma}(\delta, n, \beta, \lambda)$ reduces to the class $\mathcal{H}_{\Sigma}(n, \beta, \lambda)$ introduced by S. Porwal and M. Darus [14] and for $\delta=1, n=0$ it reduces to the class $\mathcal{H}_{\Sigma}(\beta, \lambda)$ introduced by B.A. Frasin and M.K. Aouf [9]. Also for $\delta=1, n=0, \lambda=1$ this class reduces to the class $\mathcal{H}_{\Sigma}(\lambda)$ introduced by Srivastava et. al. [17]. 
Theorem 7. Let the function $f(z)$ given by (1) be in the class $\mathcal{H}_{\Sigma}(\delta, n, \beta, \lambda)$. Then,

$$
\left|a_{2}\right| \leq \sqrt{\frac{2(1-\beta)}{(1+2 \delta)^{n}(1+2 \lambda \delta)}}
$$

and

$$
\left|a_{3}\right| \leq \frac{4(1-\beta)^{2}}{(1+\delta)^{2 n}(1+\lambda \delta)^{2}}+\frac{2(1-\beta)}{(1+2 \delta)^{n}(1+2 \lambda \delta)} .
$$

Proof. Eqns. (19) and (20) implies that there exists $p(z), q(w) \in \mathcal{P}$ such that

$$
\frac{(1-\lambda) D_{\delta}^{n} f(z)+\lambda D_{\delta}^{n+1} f(z)}{z}=\beta+(1-\beta) p(z)
$$

and

$$
\frac{(1-\lambda) D_{\delta}^{n} g(w)+\lambda D_{\delta}^{n+1} g(w)}{w}=\beta+(1-\beta) q(w)
$$

where $p(z)$ and $q(w)$ are given by (11) and (12) respectively.

Equating the coefficients in (23) and (24), we get

$$
\begin{gathered}
(1+\delta)^{n}(1+\lambda \delta) a_{2}=(1-\beta) c_{1} \\
(1+2 \delta)^{n}(1+2 \lambda \delta) a_{3}=(1-\beta) c_{2} \\
-(1+\delta)^{n}(1+\lambda \delta) a_{2}=(1-\beta) d_{1} \\
(1+2 \delta)^{n}(1+2 \lambda \delta)\left(2 a_{2}^{2}-a_{3}\right)=(1-\beta) d_{2}
\end{gathered}
$$

Using (25) and (27), we obtain

$$
c_{1}=-d_{1}
$$

and

$$
2(1+\delta)^{2 n}(1+\lambda \delta)^{2} a_{2}^{2}=(1-\beta)^{2}\left(c_{1}^{2}+d_{1}^{2}\right)
$$

Adding (26) and (28), we obtain

$$
2(1+2 \delta)^{n}(1+2 \lambda \delta) a_{2}^{2}=(1-\beta)\left(c_{2}+d_{2}\right)
$$

Or

$$
a_{2}^{2}=\frac{(1-\beta)\left(c_{2}+d_{2}\right)}{2(1+2 \delta)^{n}(1+2 \lambda \delta)}
$$

Using Lemma 3, we get

$$
\left|a_{2}^{2}\right| \leq \frac{2(1-\beta)}{(1+2 \delta)^{n}(1+2 \lambda \delta)}
$$


which gives the desired result (21).

Next, for the estimates of $\left|a_{3}\right|$, subtracting (28) from (26), we obtain

$$
2(1+2 \delta)^{n}(1+2 \lambda \delta)\left(a_{3}-a_{2}^{2}\right)=(1-\beta)\left(c_{2}-d_{2}\right)
$$

Or

$$
a_{3}=a_{2}^{2}+\frac{(1-\beta)\left(c_{2}-d_{2}\right)}{2(1+2 \delta)^{n}(1+2 \lambda \delta)}
$$

Using (30), we obtain

$$
a_{3}=\frac{(1-\beta)^{2}\left(c_{1}^{2}+d_{1}^{2}\right)}{2(1+\delta)^{2 n}(1+\lambda \delta)^{2}}+\frac{(1-\beta)\left(c_{2}-d_{2}\right)}{2(1+2 \delta)^{n}(1+2 \lambda \delta)}
$$

Using Lemma 3, we get

$$
\left|a_{3}\right| \leq \frac{4(1-\beta)^{2}}{(1+\delta)^{2 n}(1+\lambda \delta)^{2}}+\frac{2(1-\beta)}{(1+2 \delta)^{n}(1+2 \lambda \delta)}
$$

which is the desired result (22).

This completes the proof of Theorem 7 .

\section{Remarks}

Remark 8. If we put $\delta=1$ in Theorem 5 and Theorem 7, we obtain the corresponding results due to $S$. Porwal and M. Darus [14].

Remark 9. If we put $\delta=1$ and $n=0$ in Theorem 5 and Theorem 7, we obtain the corresponding results due to B.A. Frasin and M.K. Aouf [9].

Remark 10. If we put $\delta=1, n=0$ and $\lambda=1$ in Theorem 5 and Theorem 7, we obtain the corresponding results due to Srivastava et. al. [17].

\section{References}

[1] F.M. Al-Oboudi, On univalent functions defined by a generalized Sălăgean operator, Int. J. Math. and Math. sci., 27 (2004), 1429-1436.

[2] D.A. Brannan, J.G. Clunie (Eds.), Aspects of Contemporary Complex Analysis, (Proceedings of the NATO Advanced Study Institute held at the University of Durham, Durham; July 1-20, 1979), Academic Press, New York and London (1980). 
[3] D.A. Brannan, J.G. Clunie, W.E. Kirwan, Coefficient estimates for a class of starlike functions, Canad. J. Math., 22 (1970), 476-485.

[4] D.A. Brannan, T.S. Taha, On some classes of bi-univalent functions, Studia Univ. BabesBolyai Math., 31, No. 2 (1986), 70-77.

[5] S. Bulut, Coefficient estimates for a class of analytic and bi-univalent functions, Novi. Sad. J. Math., 43, No. 2 (2013), 59-65.

[6] S. Bulut, Coefficient estimates for new subclasses of analytic and bi-univalent functions defined by Al-Oboudi differential operator, J. Function Spaces and Appl., Article ID 181932 (2013), 1-7.

[7] M. Cağlar, H. Orhan, N. Yağmur, Coefficient bounds for new subclasses of bi-univalent functions, Filomat, 27, No. 7 (2013), 1165-1171.

[8] P.L. Duren, Univalent Functions, Grundlehren der Mathematischen Wissenschaften, Springer, New York (1983).

[9] B.A. Frasin, M.K. Aouf, New subclasses of bi-univalent functions, Appl. Math. Lett., 24 (2011), 1569-1573.

[10] M. Lewin, On a coefficient problem for bi-univalent functions, Proc. Amer. Math. Soc., 18 (1967), 63-68.

[11] W.C. Ma, D. Minda, A unified treatment of some special classes of univalent functions, In: Proceedings of the Conference on Complex Analysis, Tianjin (1992), 157-169.

[12] E. Netanyahu, The minimal distance of the image boundary from the origin and the second coefficient of a univalent function in $|z|<1$, Arch. Rational Mech. Anal., 32 (1969), 100-112.

[13] Ch. Pommerenke, Univalent functions, Vandenhoeck and Rupercht, Göttingen (1975).

[14] S. Porwal, M. Darus, On a new subclass of bi-univalent functions, J. Egyptian Math. Soc., 21, No. 3 (2013), 190-193.

[15] G.S. Sălăgean, Subclasses of univalent functions, In: Complex Analysis- Fifth Romanian Finish Seminar, Bucharest, 1 (1983), 362-372.

[16] H.M. Srivastava, D. Bansal, Coefficient estimates for a subclass of analytic and biunivalent functions, J. Egyptian Math. Soc., 23, No. 2 (2015), 242-246.

[17] H.M. Srivastava, A.K. Mishra, P. Gochhayat, Certain subclasses of analytic and biunivalent functions, Appl. Math. Lett., 23 (2010), 1188-1192.

[18] T.S. Taha, Topics in Univalent Function Theory, Ph.D. Thesis, University of London, London (1981). 
